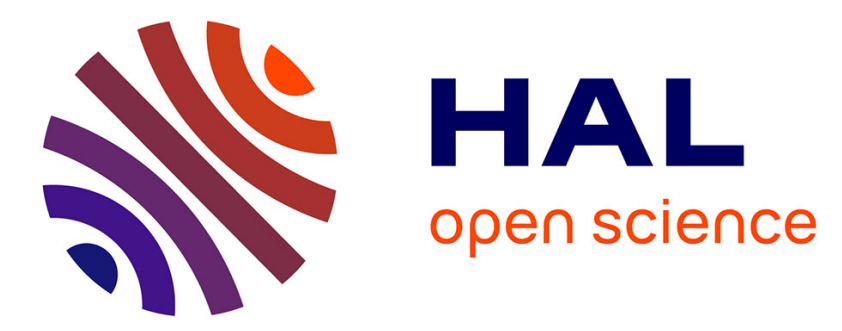

\title{
Comportement mécanique et modélisation numérique de la lamelle mitoyenne de lin
}

Karine Charlet, Brigitte Gaillard-Martinie, Alexis Beakou

\section{To cite this version:}

Karine Charlet, Brigitte Gaillard-Martinie, Alexis Beakou. Comportement mécanique et modélisation numérique de la lamelle mitoyenne de lin. 17èmes Journées Nationales sur les Composites (JNC17), Jun 2011, Poitiers-Futuroscope, France. pp.110. hal-00598102

\section{HAL Id: hal-00598102 https://hal.science/hal-00598102}

Submitted on 4 Jun 2011

HAL is a multi-disciplinary open access archive for the deposit and dissemination of scientific research documents, whether they are published or not. The documents may come from teaching and research institutions in France or abroad, or from public or private research centers.
L'archive ouverte pluridisciplinaire $\mathbf{H A L}$, est destinée au dépôt et à la diffusion de documents scientifiques de niveau recherche, publiés ou non, émanant des établissements d'enseignement et de recherche français ou étrangers, des laboratoires publics ou privés. 


\title{
Comportement mécanique et modélisation numérique de la lamelle mitoyenne de lin
}

\section{Mechanical behaviour and numerical modelling of the middle lamella of flax}

\author{
Karine Charlet $^{1}$, Brigitte Gaillard-Martinie ${ }^{2}$ et Alexis Béakou ${ }^{1}$ \\ ${ }^{1}$ Clermont Université, Institut Français de Mécanique Avancée (IFMA) / \\ Laboratoire de Mécanique et Ingénieries (LaMI), BP 10448 - F-63000 Clermont Ferrand \\ e-mail : karine.charlet@ifma.fr, alexis.beakou@ifma.fr \\ ${ }^{2}$ INRA de Clermont-Ferrand-Theix, Plateau Technique de Microscopie, \\ F-63122 Saint-Genès Champanelle \\ e-mail : brigitte.gaillard-martinie $@$,clermont.inra.fr
}

\begin{abstract}
Résumé
La modélisation du comportement en traction de composites à base de fibres de lin nécessite d'identifier les propriétés de chacun des constituants, à savoir les fibres, la matrice, l'interface fibre/matrice, mais aussi l'interface présente entre les fibres élémentaires de lin. Ce dernier est un élément difficile à caractériser mais dont les propriétés sont à considérer pour comprendre le mode de déformation et de rupture des composites dérivés. Dans cette étude, nous présentons le protocole et les résultats d'essais mécaniques et d'observations microscopiques menés à la fois sur des paires de fibres et sur des paquets de fibres. Les données recueillies sont destinées à évaluer les propriétés mécaniques de cette interface ainsi que celles des faisceaux de fibres. Ces dernières sont comparées avec de précédents travaux. En parallèle, un modèle de comportement mécanique d'interface a été développé en vue d'estimer la rigidité et la résistance de la lamelle mitoyenne, afin, par la suite, de déterminer leur influence sur le mode de déformation des faisceaux de fibres de lin puis des composites.
\end{abstract}

\begin{abstract}
Numerical modelling of the tensile behaviour of flax fibre based composites requires the identification of the properties of each constituent, not only those of the fibres, the matrix and the fibre/matrix interface but also those of the fibre/fibre interface. The latter is relatively hard to characterize but its properties have to be taken into account when trying to understand the deformation and rupture behaviours of the derived composites. In this study, the experimental protocols carried out to determine the mechanical properties and the microscopic morphology of the fibre/fibre interface and of flax bundles are described. The data obtained enables the estimation of the mechanical properties of the middle lamella as well as those of flax bundles. Whereas the latter are compared with previous literature data, the former are used to develop a numerical model of the mechanical behaviour of the fibre/fibre interface, aiming at determining its influence on the deformation mode of flax bundles and of the derived composites.
\end{abstract}

Mots Clés : lin, interface, faisceau, propriétés mécaniques, modélisation numérique Keywords : flax, interface, bundle, mechanical properties, numerical modelling

\section{Introduction}

Dans le domaine des éco-composites à fibres longues (par exemple, de lin ou de chanvre), le renfort se présente généralement sous forme de faisceaux, ou de fibres techniques, qui regroupent plusieurs fibres élémentaires collées entre elles par un ciment pectique appelé lamelle mitoyenne [1]. Alors que les propriétés mécaniques des fibres élémentaires de lin sont relativement bien connues (contrainte à rupture comprise entre 300 et $2000 \mathrm{MPa}$ [2-4], module d'élasticité variant de 25 à 120 $\mathrm{GPa}[3-5])$, celles de cette interface pectique le sont beaucoup moins. La principale explication à ce manque d'informations réside dans la difficulté à accéder à cette lamelle de quelques dixièmes de micron d'épaisseur sans altérer les fibres contiguës. De plus, les techniques classiques de caractérisation des interfaces qui créent de façon artificielle le contact entre la fibre et la matrice 
(fragmentation, pull-out...) ne peuvent pas être utilisées ici. Néanmoins, la modélisation du comportement des éco-composites requiert de connaître les propriétés mécaniques de chacun des constituants : celles des fibres naturelles, de la matrice polymère, des interfaces fibre/matrice, mais aussi celles des interfaces fibre/fibre. Ici, nous présenterons un protocole de caractérisation de la lamelle mitoyenne présente entre deux fibres de lin adjacentes ainsi qu'un modèle numérique de leur comportement basé sur un modèle de zone cohésive mis au point au laboratoire. D'autre part, des essais mécaniques menés sur des faisceaux de fibres de différentes longueurs permettront de valider leur mode de déformation et serviront de point de départ pour modéliser leur comportement en tenant compte des interfaces entre fibres.

\section{Protocole expérimental}

\subsection{Observations microscopiques}

Des observations réalisées en microscopies optique et électroniques sur plusieurs centaines de fibres de lin ont permis de déterminer les dimensions des interfaces présentes entre deux fibres (Fig. 1). Pour la microscopie optique, les faisceaux ont été enrobés dans une résine epoxy puis coupés transversalement à leur axe principal. Les images, obtenues grâce à un microscope Axioplan $2 \mathrm{E}$, ont ensuite été traitées grâce aux logiciels ImageJ et Paint Shop Pro afin d'obtenir, pour chaque couple de fibres, la largeur de leur interface commune (appelée par la suite $\ell$ ). Les résultats, traités de façon statistiques, ont permis de déterminer la largeur moyenne d'une interface en fonction de la taille des fibres impliquées dans ce contact.

En ce qui concerne l'épaisseur de ces interfaces fibre/fibre, des micrographies électroniques en transmission de faisceaux de fibres (préalablement enrobés dans une matrice époxy (Epon 812)) prises sur un Hitachi H-7650 nous ont permis d'estimer, après traitement d'images, une épaisseur moyenne de contact (appelée par la suite e).

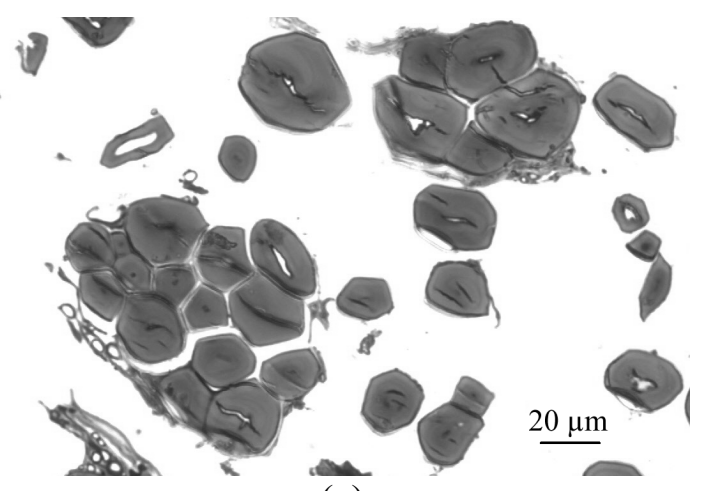

(a)

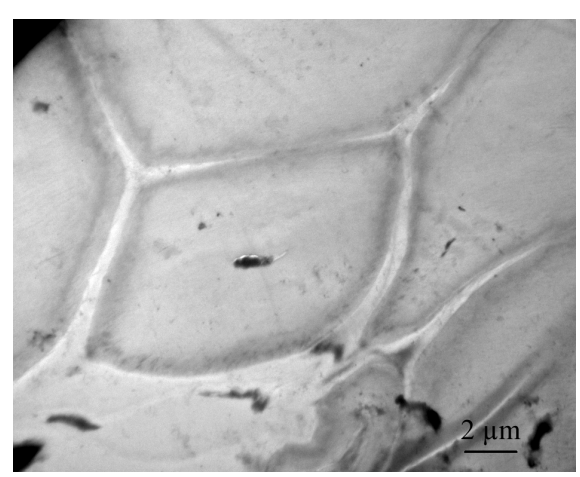

(b)

Fig. 1. Exemple de micrographies(a) optique et (b) électronique en transmission obtenues sur des sections de faisceaux de lin enrobés.

\subsection{Essai de traction sur des paires de fibres et des faisceaux}

Des essais de traction menés sur des paires de fibres contiguës ont permis d'estimer la charge maximum que pouvait supporter leur interface avant rupture. Pour cela, des paires de fibres extraites manuellement d'un ruban de lin roui, teillé et peigné, fourni par la société Safilin, ont été collées sur des cadres en carton de telle sorte que la longueur libre des échantillons soit d'environ $10 \mathrm{~mm}$. Leur configuration était telle que seule une extrémité de chaque fibre était collée sur chacun des bords du carton. Les bords supérieur et inférieur de chaque carton ont ensuite été serrés dans les mors pneumatiques d'une machine de traction Instron 5543 puis les bords latéraux coupés. Pendant l'essai, une caméra numérique (PCO Sensicam, 1.4 Mpixels) et un microscope optique (Sigma) ont été utilisés afin de valider le mode de rupture de l'échantillon. En effet, lors de la préparation, rien 
ne permettait d'être sûr d'être dans la configuration adéquate permettant de mettre en évidence le glissement des fibres et donc le cisaillement de l'interface ; des fibres pouvaient par exemple être collées sur le carton à leurs deux extrémités. Au final, sur une centaine de paires de fibres préparées, seules $30 \%$ étaient dans la bonne configuration. La prise de photos avant et après l'essai permettait également de mesurer la longueur de contact (appelée par la suite L) des fibres de l'échantillon (Fig. 2).

Les essais de traction, menés à $0,1 \mathrm{~mm} / \mathrm{min}$ jusque rupture, donnaient l'évolution de la force en fonction du déplacement imposé. Enfin, les données microstructurales établies précédemment permettaient de calculer la contrainte de cisaillement maximum $\tau$ et le module de cisaillement $\mathrm{G}$ de chaque interface testée, suivant les équations:

$$
\begin{gathered}
\tau=\frac{F_{\max }}{L \times \ell} \\
G=\frac{e \times K}{L \times \ell}
\end{gathered}
$$

avec $\mathrm{L}, \ell$ et e les dimensions de l'interface et $\mathrm{K}$ la pente initiale de la courbe force-déplacement.
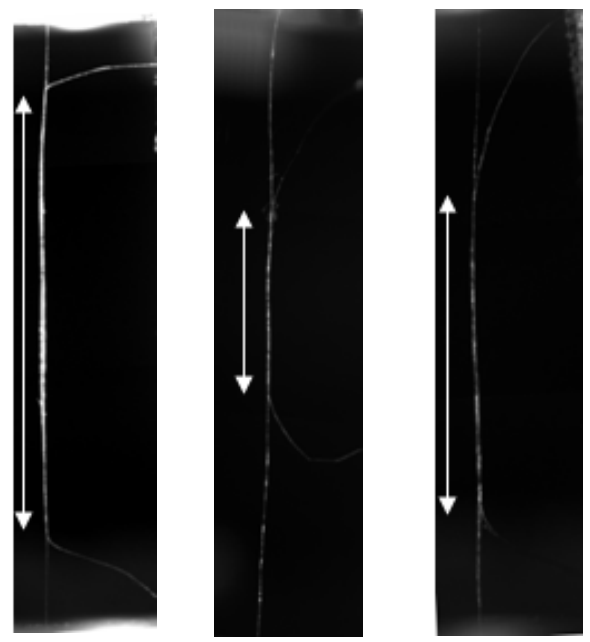

(a)

Fig. 2. Exemples de photos prises avant essai de traction sur des paires de fibres, permettant de déterminer la longueur L de contact (flèches blanches).

De la même façon, des faisceaux de plusieurs fibres ont été testés en traction, suivant le même protocole et sur la même machine, avec différentes longueurs de jauge (de $5 \mathrm{~mm}$ à $60 \mathrm{~mm}$ ). Au moins 3 faisceaux d'une longueur donnée ont été testés. Des mesures de diamètres apparents de chaque fibre du faisceau testé permettaient ensuite de tracer l'évolution de la contrainte avec la déformation, et de déterminer la contrainte maximum et la rigidité de chaque échantillon.

\subsection{Modélisation numérique de l'interface et des faisceaux de fibres.}

L'interface étant caractérisée, un modèle d'endommagement élastique a été adopté et ses paramètres identifiés à partir des résultats expérimentaux. L'épaisseur de l'interface étant très faible, le modèle d'Alfano et Crisfield [6], initialement développé pour l'étude du délaminage des composites stratifiés et qui assimile l'interface à une zone cohésive, a été adapté au cas des fibres de lin. En concordance avec la nature des essais réalisés, un mode de glissement plan au sens de la mécanique de la rupture (mode II) est retenu.

Les premières simulations numériques par éléments finis ont consisté à simuler l'essai de caractérisation afin de valider la loi d'interface programmée. Ces simulations ont été effectuées 
avec le code de calcul ANSYS. Les deux fibres élémentaires ont été modélisées par l'élément isoparamétrique SOLID95 tandis que la zone de contact est représentée par les éléments d'interface CONTA174 et TARGE170. L'option CZM, pour "cohesive zone model », a été adoptée pour prendre en compte les paramètres d'interface mesurés expérimentalement (raideur $\mathrm{K}$, contrainte de cisaillement $\tau$, glissement à rupture $\delta$ ). L'extrémité libre d'une des deux fibres est bloquée tandis qu'un déplacement longitudinal est appliqué à l'autre fibre. Après résolution du problème, la contrainte moyenne de cisaillement est tracée en fonction du glissement.

\section{Résultats}

\subsection{Dimensions des interfaces}

Grâce aux observations microscopiques de sections de faisceaux de lin, réalisées à l'aide d'un microscope optique ou d'un microscope électronique en transmission, la largeur et l'épaisseur des interfaces ont pu être estimées. La largeur $\ell$ de l'interface mesurée entre deux fibres a été corrélée aux diamètres des deux fibres en question ( $\mathrm{D}$ pour le plus grand et $\mathrm{d}$ pour le plus petit), par l'équation suivante :

$$
\ell=0,1494 \cdot d+0,1308 \cdot \mathrm{D}+4,2302
$$

L'erreur relative commise par l'utilisation de cette équation basée sur des calculs statistiques est de l'ordre de $20 \%$. Ainsi, pour une paire de fibres données dont les diamètres peuvent facilement être obtenus par microscopie optique avant essai de traction, il était possible d'évaluer la largeur de l'interface mise en jeu, généralement comprise entre 5 et $15 \mu \mathrm{m}$.

En ce qui concerne l'épaisseur de l'interface, la corrélation entre cette valeur et les dimensions des fibres impliquées dans le contact était moins évidente. Néanmoins, aux vues des données recueillies sans considérer les points triples de jonction de fibres, une épaisseur moyenne de $0,5 \mu \mathrm{m}$ a pu être estimée, en accord avec les valeurs trouvées dans la littérature [1;7].

\subsection{Propriétés moyennes, comportement mécanique et modélisation de l’interface}

Chacune des 30 courbes force-déplacement obtenues en testant en traction des paires de fibres (Fig. 3) montre une évolution quasi linéaire de la déformation de l'interface, puis une première chute de la force se produit, correspondant au début de l'endommagement de l'interface, avant la rupture finale quelques instants plus tard. Ces deux points (initiation de l'endommagement et rupture) seront utilisés par la suite pour la modélisation numérique du comportement de l'interface. La présence de deux pics et non d'un seul (comme dans le cas des fibres élémentaires) pourrait s'expliquer par le mode de «collage » des deux fibres unitaires : il serait quasi continu, comme le montrent les observations microscopiques (pas de porosité apparente dans l'épaisseur de l'interface) mais avec deux points d'accroche principaux, qui pourraient par exemple correspondre avec les extrémités des fibres, où, a priori, l'interface devrait être plus épaisse. Des observations de sections successives de faisceaux en microscopie électronique sont actuellement en cours pour permettre de valider cette hypothèse. 


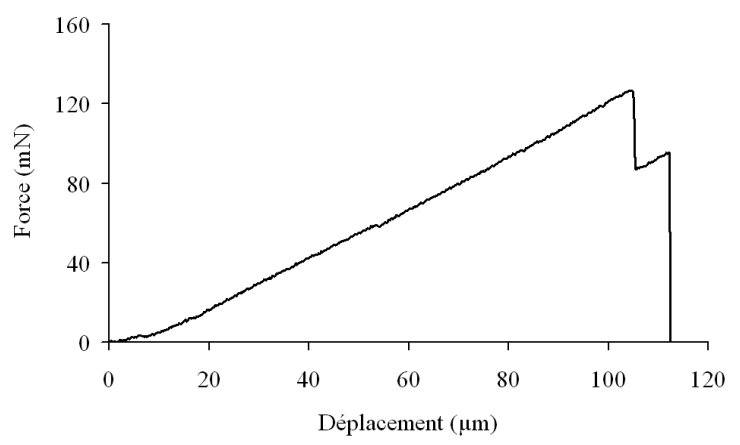

Fig. 3. Exemple de courbe force-déplacement en traction d'une paire de fibres, représentant leur glissement l'une par rapport à l'autre

En ce qui concerne les propriétés mécaniques de l'interface, les données recueillies au début de l'endommagement et l'équation (1) ont permis d'estimer la contrainte moyenne de cisaillement de l'interface à 2,9 $\mathrm{MPa}$, avec des valeurs globalement comprises entre 1 et $5 \mathrm{MPa}$. Ces valeurs sont à comparer avec celles trouvées dans la littérature pour l'interface entre des fibres de lin et des matrices polymères classiques, généralement entre 6 et $22 \mathrm{MPa}$ [8-10]. Cela explique pourquoi, jusqu'à maintenant, les propriétés des éco-composites calculées sans prendre en compte les lamelles mitoyennes étaient souvent surestimées. Cela montre également l'intérêt de diviser le plus possible les faisceaux de lin, pour diminuer la quantité d'interfaces fibre/fibre au profit des interfaces fibre/matrice souvent plus résistantes.

L'équation (2) appliquée aux raideurs déterminées à partir des courbes force-déplacement des interfaces a conduit à un module de cisaillement moyen de $19 \mathrm{kPa}$, valeur très faible comparée aux modules généralement observés pour les polymères (entre $110 \mathrm{MPa}$ pour le LDPE et $1570 \mathrm{MPa}$ pour le PMMA [11]). Par contre, c'est une valeur relativement élevée comparée aux résultats d'études rhéologiques menées sur des pectines, qui donnaient des modules de l'ordre de $1 \mathrm{kPa}$ [12;13]. Il est donc possible que la lamelle mitoyenne, principalement composée de pectines, soit également constituée d'éléments qui renforceraient sa rigidité, comme par exemple des lignines. Seules des mesures précises de la composition biochimique de cette interphase pourraient confirmer cette hypothèse.

La figure suivante (Fig. 4) présente la géométrie retenue pour modéliser par éléments finis le comportement mécanique de l'interface et la courbe contrainte-glissement obtenue. Cette dernière met en évidence un endommagement progressif de l'interface. On y retrouve la valeur de la contrainte maximale de cisaillement à partir de laquelle cet endommagement est amorcé. Toutefois, en raison de l'algorithme non-linéaire de résolution et des difficultés de convergence numérique, la raideur d'interface n'est pas bien respectée par le modèle.

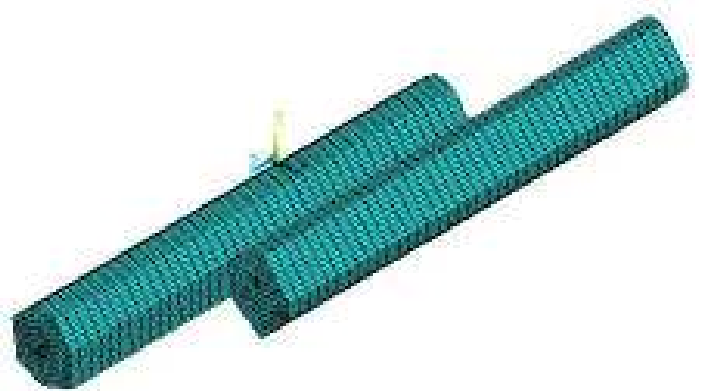

(a)

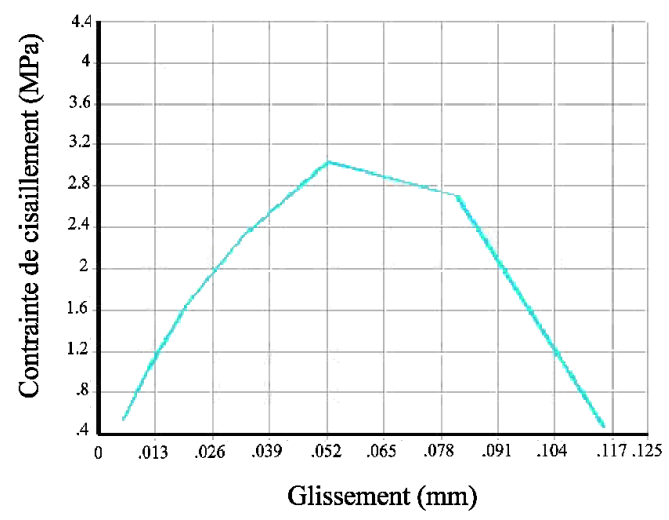

(b)

Fig. 4. Simulation de l'essai de caractérisation de l'interface : (a) maillage des fibres (gauche) et (b) courbe contrainte - glissement 


\subsection{Propriétés moyennes et comportement mécanique de faisceaux de fibres}

Les courbes force-déplacement obtenues en testant en traction des faisceaux de plusieurs fibres (Fig. 5) montrent l'influence de la longueur libre de l'échantillon sur son comportement mécanique : pour des faibles longueurs de jauge ( $a$ et $b$ ), on observe des ruptures successives de fibres. Cela est confirmé par la baisse de raideur après chaque chute de force : chaque rupture de fibre élémentaire diminue la section porteuse de la charge restante, donc le niveau de force correspondant diminue d'autant, à contrainte constante. D'un autre côté, pour de grandes longueurs de jauge (c), ces ruptures successives ne sont pas observées et l'allure de la courbe forcedéplacement ressemble à celle obtenue en testant une paire de fibres (Fig. 3). En effet, la probabilité pour qu'aucune fibre élémentaire ne soit maintenue à ses deux extrémités augmente avec la longueur de jauge, et lorsqu'elle est suffisante, seul le cisaillement des interfaces intervient.

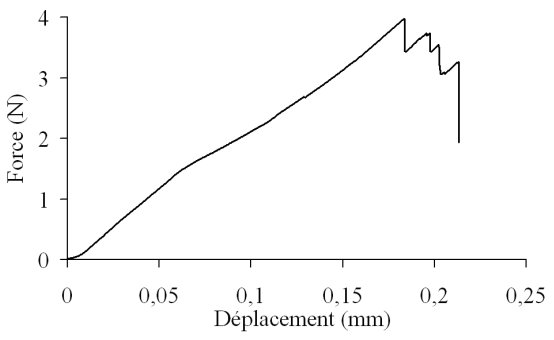

(a)

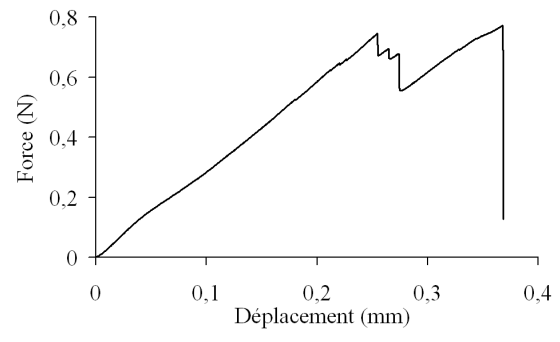

(b)

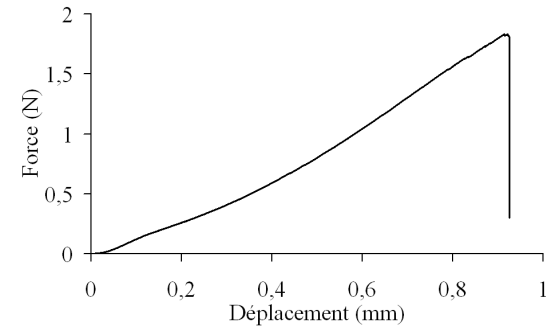

(c)

Fig. 5. Exemples de courbes force-déplacement de faisceaux de fibres à différentes longueurs de jauge: (a) $5 \mathrm{~mm}$, (b) $25 \mathrm{~mm}$ et (c) $60 \mathrm{~mm}$.

A partir de ces courbes force-déplacement et des mesures de diamètres des fibres de chaque faisceau, les contraintes maximum et les modules d'élasticité ont pu être déterminés. Leur évolution en fonction de la longueur de jauge (Fig. 6) montre une diminution dans les deux cas, avec, pour la contrainte maximum, un palier autour de $500 \mathrm{MPa}$ dès que la longueur libre des faisceaux testés dépassait environ $30 \mathrm{~mm}$. Cela peut s'expliquer par le fait que, comme dit plus haut, la probabilité qu'alors les fibres ne soient plus serrées à leurs deux extrémités dans les mors mais plutôt qu'il y ait cisaillement de leur interface est plus grande. Par opposition, aux petites longueurs de jauge, on retrouve les valeurs de contrainte à rupture des fibres élémentaires [3-5]. Entre les deux interviennent à la fois des ruptures et des glissements de fibres. Les valeurs trouvées dans la littérature [14] corroborent d'ailleurs les résultats expérimentaux de cette étude. En ce qui concerne le module d'élasticité des faisceaux, une baisse de la rigidité avec la longueur de jauge peut là encore s'expliquer par le fait qu'agissent alors les rigidités des fibres, et de plus en plus à mesure que la longueur augmente, les rigidités d'interface, plus faibles.

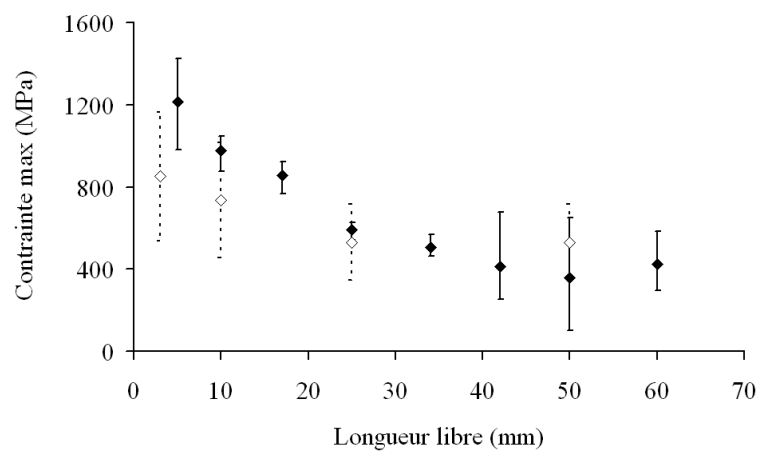

(a)

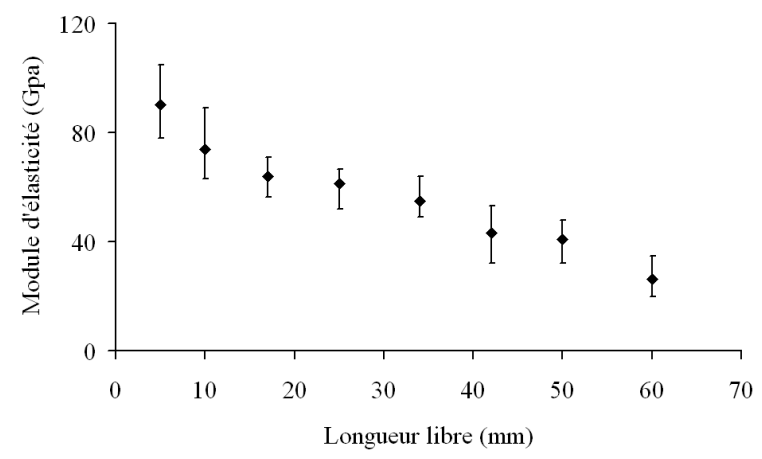

(b)

Fig. 6. (a) Contrainte maximum et (b) module d'élasticité en traction de faisceaux de fibres en fonction de la longueur libre testée (les points blancs sont les données expérimentales de Bos [14]) 
Les simulations en cours visent à étudier l'influence de la prise en compte de cette loi d'interface sur le module et la résistance d'un faisceau de plusieurs fibres.

\section{Conclusion}

L'étude du comportement mécanique de l'interface présente entre des fibres élémentaires de lin a permis de déterminer ses propriétés en cisaillement et de mettre au point un modèle numérique de déformation en adéquation avec les observations expérimentales. Ces informations sont capitales pour parvenir à modéliser correctement le comportement des composites à base de fibres de lin. En effet, ces interfaces fibre/fibre sont beaucoup moins résistantes et rigides que les interfaces entre les fibres de lin et les matrices polymères classiques. Elles sont donc non seulement à prendre en compte mais aussi éventuellement à éliminer pour obtenir des éco-composites compétitifs.

\section{Remerciements}

Les auteurs remercient le CNRS pour le financement de ce projet dans le cadre du programme Matériaux et Procédés de Substitution et de Remplacement 2010, ainsi que le Plateau Technique de Microscopie de Clermont Ferrand pour la réalisation des micrographies optiques et électroniques des sections de fibres.

\section{Références}

[1] C. Morvan, C. Andème-Onzighi, R. Girault, D.S. Himmelsbach, A. Driouich, D.E. Akin, "Building flax fibres: more than one brick in the walls", Plant Physiology and Biochemistry, Vol. 41, pp. 935-944, 2003.

[2] A. Stamboulis, C.A. Baillie, T. Peijs, "Effects of environmental conditions on mechanical and physical properties of flax fibers", Composites Part A, Vol. 32, pp. 1105-1115, 2001.

[3] C. Baley, "Analysis of the flax fibres tensile behaviour and analysis of the tensile stiffness increase", Composites Part A, Vol. 33, pp. 939-948, 2002.

[4] J. Andersons, E. Spārniņš, R. Joffe, L. Wallström, "Strength distribution of elementary flax fibres", Composites Science and Technology, Vol. 65, pp. 693-702, 2005.

[5] K. Charlet, C. Baley, C. Morvan, J.P. Jernot, M. Gomina, J. Bréard, "Characteristics of Hermès flax fibres as a function of their location in the stem and properties of the derived unidirectional composites", Composites Part A, Vol. 38, pp. 1912-1921, 2007.

[6] G. Alfano, M.A. Crisfield, "Finite element interface models for the delamination analysis of laminated composites: mechanical and computational issues", International Journal for Numerical Methods in Engineering, Vol. 50, pp. 17011736, 2001.

[7] B. Domengès, K. Charlet, "Direct Insights on Flax Fiber Structure by Focused Ion Beam Microscopy", Microscopy and Microanalysis, Vol. 16, pp. 175-182, 2010.

[8] A. Stamboulis, C. Baillie, E. Schulz, "Interfacial characterization of flax fibre-thermoplastic polymer composites by the pull-out test", Die Angewandte Makromolekulare Chemie, Vol. 272, pp. 117-120, 1999.

[9] N.E. Zafeiropoulos, C.A. Baillie, F.L. Matthews, "Modification and characterization of the interface in flax/polypropylene composite materials", Proceedings of ECCM9, Brighton, GB, June 2000.

[10] A. Le Duigou, P. Davies, C. Baley, "Interfacial bonding of Flax fibre/Poly(l-lactide) bio-composites" Composites Science and Technology, Vol. 70, pp. 231-239, 2010.

[11] E.J. Parry, D. Tabor, "Pressure dependence of the shear modulus of various polymers", Journal of Materials Science, Vol. 9, pp.289-92, 1974.

[12] F. Capel, T. Nicolai, D. Durand, P. Boulenguer, V. Langendorff, "Calcium and acid induced gelation of (amidated) low methoxyl pectin”, Food Hydrocolloid, Vol. 20, pp. 901-907, 2006.

[13] H.U. Endress, C. Döschle-Volle, K. Dengler, "Rheological methods to characterize pectins in solutions and gels", in: J. Visser, A.G.J. Voragen (Eds), Pectins and pectinases, Progress in Biotechnology, Elsevier, 1995, pp. 418.

[14] H.L. Bos, "The potential of flax fibres as reinforcement for composite materials", PhD thesis, Eindhoven, 2004. 